\title{
Phase field modeling of excimer laser crystallization of thin silicon films on amorphous substrates
}

\author{
C. J. Shih, C. H. Fang, and C. C. Lu \\ Central Research Institute, Chunghwa Picture Tubes, Ltd., 1127 Hopin Road, Padeh City, \\ Taoyuan 334, Taiwan \\ M. H. Wang \\ Institute of Microbiology and Biochemistry, National Taiwan University, Taipei 10617, Taiwan \\ M. H. Lee and C. W. Lan ${ }^{\text {a) }}$ \\ Department of Chemical Engineering, National Taiwan University, Taipei 10617, Taiwan
}

(Received 30 November 2005; accepted 24 May 2006; published online 6 September 2006)

\begin{abstract}
Excimer laser crystallization processing of thin silicon films on amorphous silicon oxide substrates was simulated by means of phase field modeling. The quantitative phase field model was derived from the Gibbs-Thompson equation coupled with energy conservation. Because the adaptive mesh scheme was adopted, the present calculations could accommodate both two-dimensional superlateral growth (SLG) phenomena and the realistic interface thickness (in the order of $10^{-10} \mathrm{~m}$ ). The vertical growth of fine-grained nucleation structures was simulated using one-dimensional calculations, and the results are consistent with those obtained in previous experiments. Two cases of SLG were also simulated, and the evolution of the interface and thermal fields was determined. Based on our simulation results, we conclude that SLG crystallization does not achieve steady growth because of the extremely fast heat dissipation from the substrate. To obtain very uniform electric characteristics for device fabrication, the layout design and the device position should take the SLG laser mask into consideration. (C) 2006 American Institute of Physics.
\end{abstract}

[DOI: $10.1063 / 1.2245193$ ]

\section{INTRODUCTION}

Over the last few decades, excimer laser crystallization has emerged as one of the most powerful processes for fabricating crystalline silicon films on amorphous substrates. Due to the high absorption coefficient of silicon at the specific wavelengths of excimer lasers, both melting and resolidification can proceed extremely rapidly so that the manufacturing of silicon on insulator (SOI) devices at low temperatures can be realized. The thin film transistors (TFTs) constructed on the poly-Si active layer in liquid crystal displays (LCDs) are a well-known application, where better crystalline silicon films can enable smaller devices and faster operating frequencies. With this processing technique, the fabrication of a full system on glass (SOG) is also possible. ${ }^{1}$

The fabrication of poly-Si films via secondary recrystallization through multishot excimer laser irradiation has been widely adopted in practical poly-Si TFT-LCD processing, ${ }^{2}$ where the optimized laser energy density (ED) is usually slightly lower than the complete melting threshold (CMT). ${ }^{3}$ Although devices with uniform electric characteristics can be obtained by using this method, the field effect mobility $\left(\mu_{\mathrm{FE}}\right)$ of carriers is still about one order smaller than that of carriers on epitaxial $\mathrm{Si}$ film because of the smaller grain size (the average size is only about five times the film thickness). Trap states near grain boundaries and defects are the major barrier to reducing the performance of poly-Si devices. ${ }^{2}$ Recently,

\footnotetext{
a) Author to whom correspondence should be addressed; electronic mail: cwlan@ntu.edu.tw
}

laser crystallization methods based on the superlateral growth (SLG) phenomena ${ }^{4}$ have been developed to improve both electric characteristics and throughput in production. ${ }^{5-8}$ The aim of these techniques is to completely melt the irradiated region in one laser shot $(\mathrm{ED} \gg \mathrm{CMT})$, while the neighboring nucleates act as seeds for growing large grains laterally. ${ }^{5}$ These nucleates may be induced by means of explosive crystallization, ${ }^{9}$ solid-phase crystallization, or fine grains formed by the lower laser fluence at the edge of SLG. These methods generally involve an optical design of the laser beam, ${ }^{5,7}$ an additional photolithographic architecture, ${ }^{8}$ a capping antireflective layer, ${ }^{6}$ or a special thermal insulation arrangement, ${ }^{8}$ which often requires much work on design and experimentation. On the other hand, to improve the quality of crystalline silicon films, controlling the propagation of interface and heat flux during crystallization is critical, and an efficient and quantitative simulation method is greatly needed.

For the simulation of laser induced melting and solidification of materials, many nonequilibrium models (i.e., where the interface temperature is far from the melting point, and materials may exist highly supercooling or superheating) ${ }^{10-15}$ have been proposed. Simulations of nucleation ${ }^{12,15}$ and explosive crystallization with double moving fronts ${ }^{13}$ have also been conducted. However, to solve the problem of free boundary movement, these models are all based on the enthalpy model ${ }^{16}$ and they only introduce an equation of thermal transport. The interface position has to be predicted by an additional tracker, which requires information about the solidification speed and local temperature so that the effects 
of interface properties, surface tension, and anisotropy cannot be considered. Furthermore, extending these codes to two-dimensional (2D) or three-dimensional (3D) simulations is quite difficult.

The phase field model ${ }^{17-20}$ (PFM) based on the nonequilibrium thermodynamics has emerged as a promising and powerful tool for simulating free boundary problems with complex morphological evolution. The most important feature of this model is that it treats the interface as a diffusive one having a finite thickness $\delta$. In principle, it can simulate extreme nonequilibrium solidification problems without any fitting of experimental data, such as supercooling and solute trapping. ${ }^{21}$ Moreover, because the transport equations and the phase field are solved simultaneously, the effects of surface tension, nonequilibrium, and anisotropy can be easily included. More importantly, the extension of the model to 2D and 3D simulations is straightforward. However, the drawback of PFM lies in the fact that that the minimum mesh size has to be smaller than the interface thickness $\delta$. But a realistic interface thickness is only on the order of the capillary length (approximately several angstroms). When both the overall morphology (taking the dendritic growth as an example, $\sim 1 \mathrm{~mm}$ ) and the local interface are taken into account, the huge difference in the length scales makes the simulation very challenging even with today's supercomputers. Thus, several physical and numerical approaches have been proposed to reduce the computational load. These include thin interface limit analysis (using a thicker $\delta$ to achieve quantitative results) ${ }^{18-20}$ and adaptive mesh refinement (AMR). ${ }^{22,23}$

Fortunately, when the excimer laser crystallization of thin silicon films is simulated, because of the extremely short laser pulse duration $(\sim 100 \mathrm{~ns})$, the heat transfer distance is rather small and the crystalline size is only several times the Si film thickness. Therefore, a realistic interface thickness can be adopted without too much computational effort. In other words, to solve this problem, phase field methodology is the best choice among the available approaches. Very recently, La Magna et al. ${ }^{24}$ used the one-dimensional (1D) PFM for studying the activation process of dopants following excimer laser irradiation, and the achieved solute distribution showed good agreement with experimental results. Two-dimensional cases that consider the excimer laser annealing of metal-oxide semiconductor (MOS) devices and laser light scattering have also been studied. ${ }^{25,26}$ However, the detailed evolutions of the interfaces and thermal transport were not yet studied quantitatively in this research, and the feasibility of the phase field model was not clearly verified.

In this study, we adopted the phase field model derived from the geometrical description ${ }^{17}$ and focused on the rapid melting and crystallization of a thin silicon film on an amorphous silicon oxide. The adaptive mesh refinement scheme based on the finite volume method ${ }^{23}$ was applied efficiently in $2 \mathrm{D}$ calculations. To make one-to-one comparisons with previous experimental studies, ${ }^{3,4}$ we considered a stack of amorphous silicon $(a-\mathrm{Si})$ and silicon oxide films on a single crystalline silicon wafer. Firstly, we simulated the vertical regrowth (one-dimensional computation) of fine grains and nucleation under various energy densities. Then, to examine the performance in 2D calculations, two samples related to the SLG phenomena ${ }^{4}$ were simulated, including one that involved repeatable crystallization units with the sequential lateral solidification (SLS) technique. ${ }^{5}$ The evolution of thermal fields and solid/melt interfacial fronts was also investigated. In Sec. II, the model and the adaptive method used in the simulations are briefly described. Section III presents results and discussion. Conclusions are drawn in Sec. IV.

\section{MATHEMATICAL AND PHYSICAL MODELS}

In the phase field model, the rapid melting and solidification of a thin silicon film are described by a phase field variable $\phi$ and thermal field $T$. Based on the assumption that $\phi=0$ in the solid and $\phi=1$ in the melt, the variation of this variable, defined as a hyperbolic function in the normal coordinate $n$, changes rapidly from 0 to 1 at the interface,

$$
\phi=\frac{1}{2}\left[1+\tanh \left(\frac{n}{2 \delta}\right)\right] .
$$

Following the geometrical formulation proposed by Beckermann et al., ${ }^{27}$ we can define the unit normal vector $\mathbf{n}$, the local curvature $\kappa$, and the interface speed $V_{n}$ at the interface in the direction of $\mathbf{n}$ as $\mathbf{n}=\nabla \phi /|\nabla \phi|, \kappa=\nabla \cdot \mathbf{n}=1 /|\nabla \phi|\left(\nabla^{2} \phi\right.$ $-\nabla \phi \nabla|\nabla \phi| /|\nabla \phi|)$, and $V_{n}=-1 /|\nabla \phi| \partial \phi / \partial t$. By substituting them into the generalize Gibbs-Thompson (GT) equation (for a material having isotropic surface energy $\sigma$ and a kinetic coefficient $\mu$ ), i.e.,

$$
T_{i}=T_{m}\left(1+\kappa \frac{\sigma}{L}\right)-\frac{V_{n}}{\mu},
$$

we can obtain a phase field equation,

$$
\begin{aligned}
\frac{\partial \phi}{\partial t}= & \frac{\sigma T_{m} \mu}{L}\left[\nabla^{2} \phi-\frac{\phi(1-\phi)(1-2 \phi)}{\delta^{2}}\right] \\
& -\mu\left(T_{m}-T\right)\left[\frac{\phi(1-\phi)}{\delta}\right],
\end{aligned}
$$

where $L$ is the heat of fusion and $T_{m}$ the melting point of the bulk material.

The dependence of the interface velocity on the temperature is shown in Eq. (2). Here, the kinetic effect is presented in a linear form. Other physical models, for example, the Fulcher-Vogel model, ${ }^{28}$ can also be easily adopted. The present phase field model is completely consistent with those based on nonequilibrium thermodynamics. It has been shown that if $\phi(1-\phi) / \delta$ in the last term of Eq. (3) is replaced by $5\left[\phi^{2}(1-\phi)^{2} / \delta\right]$, the present model is equivalent to Karma and Rappel's standard model. ${ }^{18}$

The heat transfer equation can be easily derived from Fourier's law, and the conservation of internal energy as follows:

$$
\rho(\phi) C(\phi) \frac{\partial T}{\partial t}=\nabla \cdot[K(\phi) \nabla T]-p^{\prime}(\phi) L \frac{\partial \phi}{\partial t}+S(d, t) .
$$

Here $\rho$ is the density, $K$ is the thermal conductivity, $C$ is the specific heat capacity, $p(\phi)$ is the interpolation function describing the diffusive interface, and the source term $S(d, t)$ 

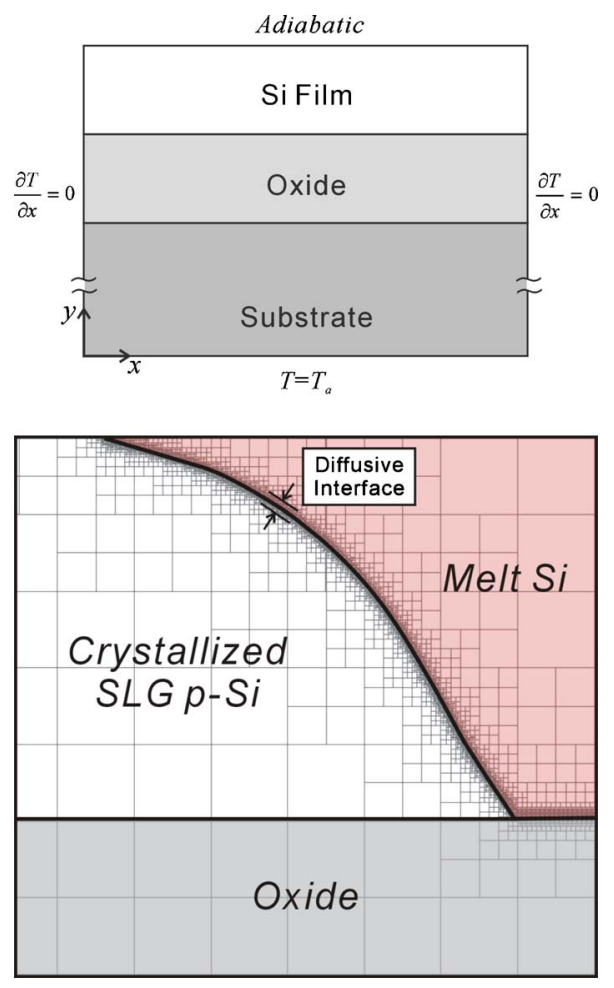

FIG. 1. (Color online) (a) Schematic diagram of the computational domain for the phase field modeling of laser crystallization in thin silicon film $(100 \mathrm{~nm})$ on a silicon oxide $(100 \mathrm{~nm}) /$ silicon wafer substrate; (b) a sample adaptive finite volume mesh with propagation of the SLG crystal/melt interface.

for the material, having a constant absorption coefficient $\alpha$, can be written as

$$
S(d, t)=(1-R) P(t) \alpha \exp (-\alpha d),
$$

where $R$ is the reflectivity of the sample, which is assumed to be a function of the temperature and material surface. Also, $d$ is the distance from the sample surface and $P(t)$ is the timedependent intensity of the laser pulse, which is set as a perfect Gaussian distribution with a full width at half maximum (FWHM) of $30 \mathrm{~ns}$.

It is worth noting that because the source term in Eq. (3) only acts inside the diffusive interface, the present methodology cannot be used to simulate the emergence of an interface from the bulk solid material $(\phi=0$ in the overall computational domain). Therefore, in this study the thermal equation was solved first for the solid phase until the surface temperature reached its melting point. Then, an artificial liquid spot ( $1 \mathrm{~nm}$ in size for the hypertangent phase field function) was imposed at the hottest surface. To test the feasibility of this approach, various spot sizes $(0.5-2 \mathrm{~nm})$ were used, and the overall melting and crystallization processes were almost the same.

A stack of $100 \mathrm{~nm}$ amorphous silicon and $100 \mathrm{~nm}$ silicon oxide films on a single crystalline silicon wafer substrate at room temperature $\left(25^{\circ} \mathrm{C}\right)$ was considered in the simulation. To minimize the far field boundary effect of the thermal transport, a thick substrate depth was considered, and the computational domain was as illustrated in Fig. 1(a). The heat dissipation through thermal convection and radiation from the surface was neglected; i.e., the adiabatic boundary condition was used at the top boundary, due to the extremely short laser purse duration. For the other boundaries, symmetric and Dirichlet boundary conditions were used. Because a large domain up to several microns was considered, to keep the smallest mesh size from being smaller than the interface thickness ( $4 \AA$ was used here), the adaptive finite volume method $^{23}$ (FVM) was used to discretize the governing equations. A sample mesh near the crystal/melt diffusive interface is illustrated in Fig. 1(b); the solid line is located at $\phi=0.5$. As shown in the figure, the AMR technique could refine grids at the diffusive interface such that smallest cell size was less than the interface thickness. Such mesh refinement was performed dynamically and concurrently with the propagation of the interfaces and the steep thermal gradients. Grid coarsening was done in a similar manner at places that were no longer of interest. For an overall 2D domain of several microns, the maximum number of finite volumes was about 60000 , depending on the complexity of the interfaces, and the numerical time step for integration was set at $10^{-3} \mathrm{~ns}$. All the calculations were performed efficiently using one personal computer (Pentium-4 3G with 512 Mbytes of DDR DRAM), and the calculation time was several days for a $2 \mathrm{D}$ case.

In many previous experiments, basic transformation scenarios showed that the grain structures of laser recrystallized Si film could be categorized as fine grains (FGs), SLG, and nucleation (NUC) regimes, depending on the applied energy densities. ${ }^{3,4}$ In the low energy density regime, the silicon film was partially melted, while the FG structures grew vertically such that the grain size was on the order of the film thickness. When the energy density exceeded CMT, homogeneous and heterogeneous nucleations were triggered as the degree of supercooling exceeded the margin of the metastable zone, ${ }^{12,15}$ and SLG took place within a narrow window near CMT. In general, with a commercial excimer laser system, one can easily observe the coexistence of these three structures at the edge of the laser beam, where the maximum ED is higher than CMT, following one-shot irradiation, as shown in Fig. 2.

To model these phenomena using our present PFM, some physical parameters were roughly tuned as shown in Table I, and other parameters not listed in the table were those adopted in Ref. 24. The interfacial tension of the oxide/ molten-Si and oxide/ $p$-Si interfaces was set to be the same. In addition, nucleation growth was treated as resolidification from the molten-Si/oxide interface with a triggering temperature $T_{\mathrm{NUC}}$ of $1300 \mathrm{~K},{ }^{15}$ where $120 \mathrm{~K}$ supercooling occurred as compared with the melting point of amorphous silicon. The classical nucleation theory was not considered here. This approach implies that only heterogeneous nucleation was involved in one-dimensional cases, while typically it is indeed the predominant mechanism. However, for two-dimensional modeling, the present method for nucleation is oversimplified. Accordingly, the present study mainly focused on the 2D SLG phenomena, and nucleation was just viewed as a "stopper" of it. In other words, our SLG simulation was not much affected by the nucleation model used. 


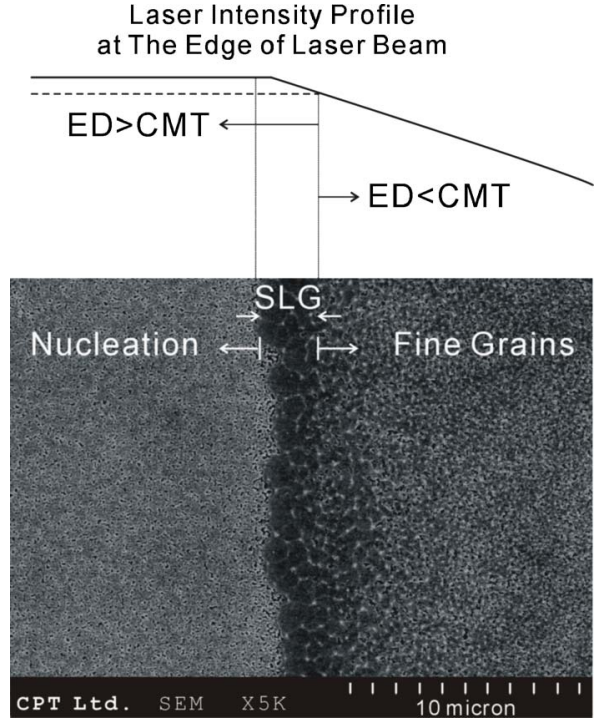

FIG. 2. Experimentally observed fine-grains, superlateral growth grains, and nucleation structures (following Secco solution etching) at the edge of the line beam laser irradiation region.

\section{RESULTS AND DISCUSSION}

\section{A. 1D simulations of FG and NUC poly-Si growth}

To determine the basic transient behavior of phase transformation and thermal fields, we simulated the vertical melting and regrowth of a silicon film under various laser energy densities. The 1D simulations were performed efficiently using our AMR scheme, and the calculation time was only about $2 \mathrm{~h}$ for each case. Because curvature does not exist in the $1 \mathrm{D}$ configuration, the kinetic effect dominates the interface temperature as shown in Eq. (2). We extracted simulated interface temperatures and speeds (all defined at $\phi=0.5$ ) to check if the phase field could correctly describe the kinetic effect. According to our results, the simulated interface temperatures and the ones from the Gibbs-Thompson equation

TABLE I. Physical parameters used in the simulations.

\begin{tabular}{lcc}
\hline \hline Density $(\rho)$ & $a-\mathrm{Si}, p-\mathrm{Si}, l-\mathrm{Si}$ & $2.33 \mathrm{~g} / \mathrm{cm}^{3}$ \\
Enthalpy $(L)$ & $a-\mathrm{Si}$ & $1320 \mathrm{~J} / \mathrm{g}$ \\
& $p-\mathrm{Si}$ & $1780 \mathrm{~J} / \mathrm{g}$ \\
Equilibrium melting point $\left(T_{m}\right)$ & $a-\mathrm{Si}$ & $1420 \mathrm{~K}$ \\
& $p-\mathrm{Si}_{(\mathrm{FG}, \mathrm{NUC})}$ & $1500 \mathrm{~K}$ \\
& $P-\mathrm{Si}(\mathrm{SLG})$ & $1690 \mathrm{~K}$ \\
Thermal conductivity $(k)$ & $a-\mathrm{Si}$ & $0.05 \mathrm{~J} /(\mathrm{scm} \mathrm{K})$ \\
& $p-\mathrm{Si}$ & $0.2 \mathrm{~J} /(\mathrm{scm} \mathrm{K})$ \\
& $\mathrm{Oxide}$ & $k(T)$ \\
Reflectivity $(R)$ & $a-\mathrm{Si}$ & 0.58 \\
& $l-\mathrm{Si}$ & 0.72 \\
Absorption coefficient $(\alpha)$ & $a-\mathrm{Si}$ & $2 \times 10^{6}$ \\
\hline \hline
\end{tabular}

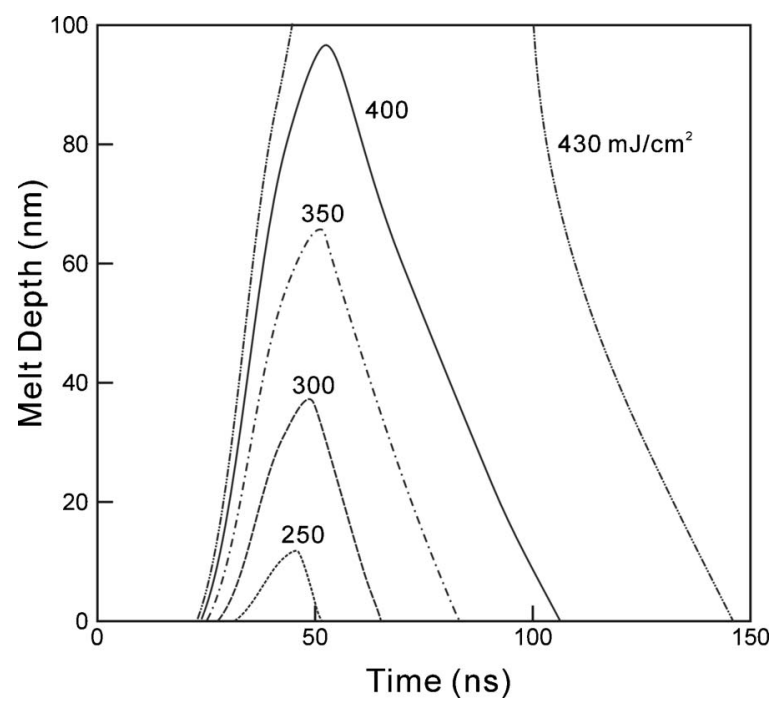

FIG. 3. Calculated positions of the solid/liquid interface (defined at $\phi$ $=0.5$ ) under various energy densities.

matched quite well, and the deviation was less than $3 \mathrm{~K}$. If finer meshes were adopted, we could obtain more accurate results. Nevertheless, for typical laser crystallization, due to the high-speed moving fronts, such deviation is negligible for energy conservation.

The evolution of the interface position and temperature under several one-shot laser energy densities is plotted in Figs. 3 and 4, respectively. As shown, when the laser intensity was high enough to reach the melting point of $a$-Si at the surface, the interface started to melt and the energy increment overcame the latent heat to move the interface. Because of the fast increase in the fluence, the interface temperature and moving velocity also increased, raising the rates of melting and thermal diffusion. When the laser intensity reached the maximum (at $30 \mathrm{~ns}$ ) value and then decayed, both the interface temperature and the melting speed also decreased. On the other hand, the interface moving velocity never reached a steady state during the melting process.

If the energy density was not sufficient to totally melt the $a$-Si film (in the present cases, $\mathrm{ED}<410 \mathrm{~mJ} / \mathrm{cm}^{2}$ ), once the

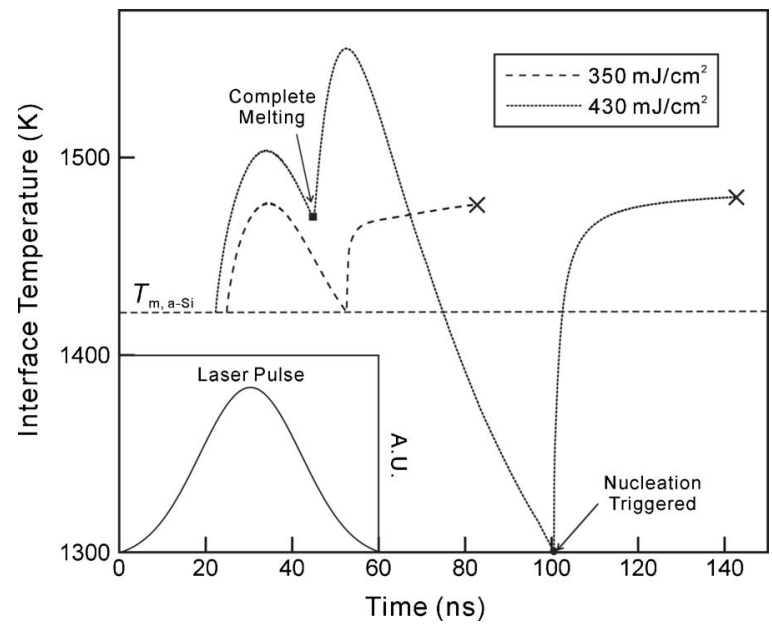

FIG. 4. Calculated interface temperatures with time under ED $=350 \mathrm{~mJ} / \mathrm{cm}^{2}(<\mathrm{CMT})$ and $\mathrm{ED}=430 \mathrm{~mJ} / \mathrm{cm}^{2}(>\mathrm{CMT})$; the regrowth of FG and NUC structures is illustrated. 


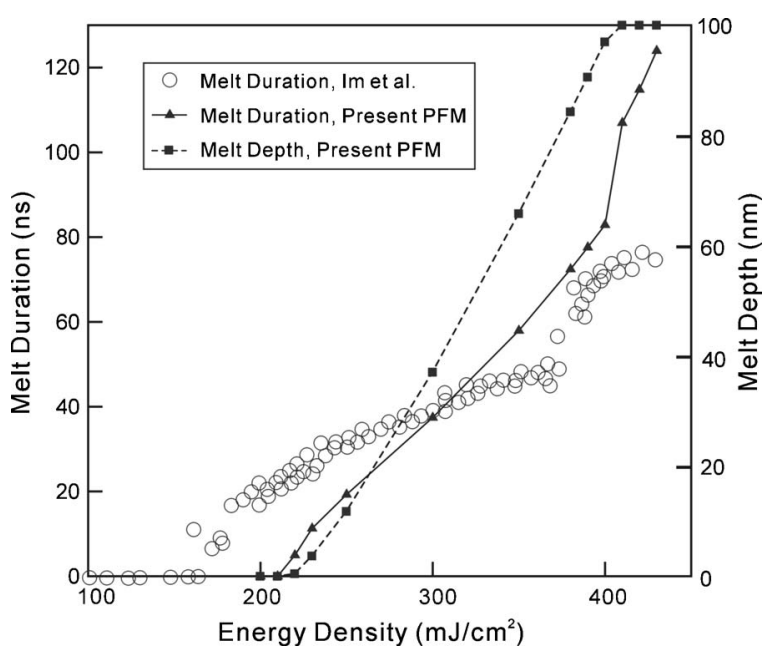

FIG. 5. Comparison of the simulated melt duration and melt depth with experimental results reported in Ref. 3.

interface temperature fell below the melting point, vertical growth of the FG structure began. Due to the linear kinetic effect term in Eq. (2), the interface temperature increased dramatically and approached steady-state solidification. According to our simulation results, the crystallization velocity decreased gradually with time and was between 2.5 and $1.5 \mathrm{~m} / \mathrm{s}$. For NUC growth, as mentioned above, the only difference comparing with FG growth was the different triggering temperature $(1300 \mathrm{~K})$, instead of $T_{m, a-\mathrm{Si}}$. Once the $a$-Si film was totally melted, the induced laser fluence did not absorb the heat of fusion, so that the interface temperature suddenly increased, as shown in Fig. 4. Following the pulse duration (at $60 \mathrm{~ns}$ ), heat dissipation from the substrate caused the interface temperature to drop quickly $(\phi=0.5$, at the silicon/oxide interface). Compared with FG growth, the temperature decreased at the end of the pulse duration instead of reaching the maximum; moreover, NUC was triggered at a large supercooling of $120 \mathrm{~K}$. In short, both factors caused a sudden increase in the melt duration, and the FGs earlier crystallized could act as seeds for SLG, as shown in Fig. 2.

A comparison of the simulated pulse duration and melt depth was made through experiments by $\operatorname{Im}$ et al., ${ }^{3}$ and the results are shown in Fig. 5. As shown in the figure, once the laser intensity was high enough to melt $a-\mathrm{Si}$, the pulse duration increased with the energy density, and a sudden rise (about $20 \mathrm{~ns}$ ) occurred due to the transformation from the FG to NUC growth mechanisms. In general, the present study showed a very similar trend with the experimental results. The deviation can be explained as follows.

(1) The physical properties of the amorphous, FG, NUC, and melt phases were not carefully tuned. After several tests, we found that the reflectivity and thermal conductivity of $a$-Si dominated the melting process, while the conductivity of the recrystallized material significantly influenced the solidification process.

(2) The present PFM only considered the coupled relation of the interface and heat transfer, while many other physical phenomena, such as explosive crystallization,
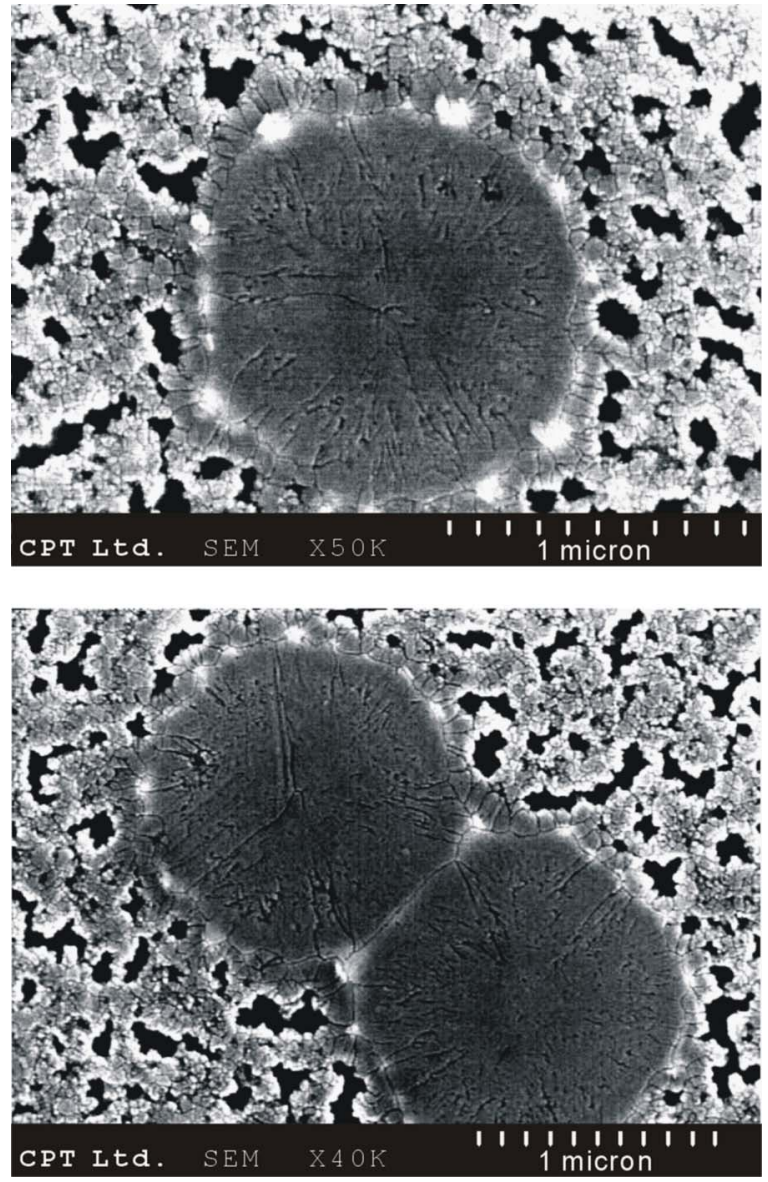

FIG. 6. Experimental observation of (a) an isolated disklike SLG grain and (b) the meeting of two neighboring grains (following Secco solution etching).

heat radiation from the surface, and nucleation phenomena, were not included. ${ }^{12,13,15}$

Indeed, most of the physical phenomena can be integrated into the PFM, and a set of best-fitted parameters can be obtained after some computational trials. For the present model, as explained in the previous analysis, we have verified that it is possible to simulate reasonably well the melting and solidification processes by using our PFM. In the next section, 2D SLG simulations will be discussed.

\section{B. 2D simulations of SLG phenomena}

As illustrated in the previous section, due to the relatively slow growth speed of vertical crystallization $(\sim 2 \mathrm{~m} / \mathrm{s})$, large grain growth often requires multishot irradiation, which is not suitable for the mass production of large $p$-Si films. ${ }^{2}$ Recently, many methods based on the superlateral growth phenomena have been proposed for SOG applications and high-throughput production. $\mathrm{Im}$ and $\mathrm{Kim}^{4}$ researched on laser-induced SLG. They reported that with a one-shot laser intensity slightly above CMT, large disklike grains were observed. We also easily obtained this interesting structure in our experiments, as shown in Fig. 6; both isolated grains (a) and the meeting of two grains (b) were clearly captured. Evidently, the size of the SLG disks totally depends on the time at which the surrounding NUC growth 

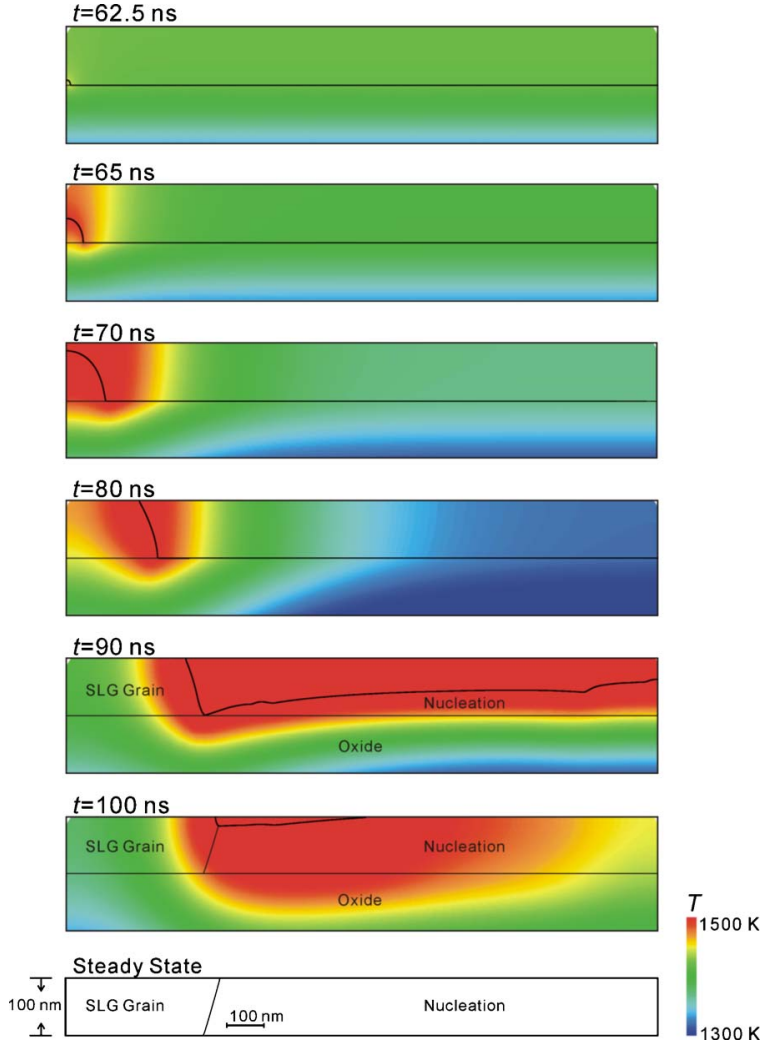

FIG. 7. (Color online) Evolution of simulated thermal fields and interfaces $(\phi=0.5)$ of disklike SLG grain growth under $\mathrm{ED}=410 \mathrm{~mJ} / \mathrm{cm}^{2}$. Note that the $y$ direction is rescaled.

is triggered. These large grains grow from surviving seeds at the bottom of the silicon film, which are formed by the explosive crystallization during the initial stage of the melting process. $^{4}$

Here, we considered the same stack of films $(100 \mathrm{~nm}$ $a$-Si/100 nm silicon oxide) on a silicon wafer at $25^{\circ} \mathrm{C}$ as that studied in Ref. 4. For disklike grain growth, because solidification proceeded laterally, while heat transfer mainly proceeded vertically, we adopted $2 \mathrm{D}$ axisymmetric discretization to resolve the highly nonlinear behavior. In fact, for isolated grain growth, this was indeed a good approach; however, if we took into account the meeting of adjacent grains, as shown in Fig. 6(b), then 3D modeling had to be performed. For phase field modeling, this requires extensive computation even with the AMR scheme, and parallel computing has to be considered. ${ }^{29}$

The evolution of interfaces and thermal fields $(\phi=0.5)$ is plotted in Fig. 7. During the initial stage, we defined a small seed on the silicon/oxide interface artificially. As the temperature at the interface dropped below the melting temperature of $a$-Si (see Fig. 4), crystallization started. Furthermore, nucleation was also defined as the growth proceeding from the bottom of the Si film, where the triggering point $T_{\mathrm{NUC}}$ was $1300 \mathrm{~K}$. As shown in Fig. 7, during the growth of a SLG grain, since a large amount of heat of fusion was released, the material was always "hot" near the interface. With the propagation of the solidification front, the heat flux became normal to the interface and then sank in the substrate beyond the thermal boundary layer. Meanwhile, when the far-field
Si/oxide interface temperature fell below $T_{\mathrm{NUC}}$, rapid heterogeneous nucleation growth began, and the SLG grain growth was finally stopped by the nucleation phase. According to our PFM results, at the onset of nucleation, no solidification occurred inside the thermal boundary layer ahead of the interface. However, nucleation was introduced by neighboring solids, and it proceeded vertically, while the surrounding sub-SLG region [see Fig. 6(a) and Ref. 4] was not observed. This is also because we treated the nucleation phase as a bulk solid and because the effect of homogeneous nucleation was not considered. In reality, the growth inside the thermal boundary layer will initially be "pulled up" by the driving force of surface tension; therefore, although the temperature will be higher than $T_{\mathrm{NUC}}$, solidification will also be triggered. As a result, it is difficult to define a sub-SLG region induced by the surrounding nucleation. The simulated disklike grain was $0.8 \mu \mathrm{m}$ in size, which is consistent with the experiment results $(0.8-1 \mu \mathrm{m}){ }^{4}$

The main purpose of investigating SLG phenomena is to better control it artificially. ${ }^{30}$ The proposed SLS technique ${ }^{5}$ has potential for both large-grain crystallization and highthroughput production. The patterned mask consists of an array of narrow slits with a specific shape such that only a small portion of the laser beam can pass through the slit to the substrate. In a commercial SLS system, rectangular slits only a few microns wide can be adopted, and long SLG grains can be obtained by overlapping these masks sequentially. In our simulation, we considered a single laser shot passing through a $2 \mu \mathrm{m}$ slit on $a$-Si film; the laser beam profile applied to the material used in our simulation (a) and a sample of the experimentally observed grains (b) are shown in Fig. 8.

To grow large grains from nucleates in the unmelted lateral portion of a film, the operating laser intensity is always kept much higher than CMT. ${ }^{5}$ In general, longer lateral growth length can be obtained with higher laser energy densities and thicker silicon films. ${ }^{15}$ In the present $100 \mathrm{~nm}$ $a$-Si/100 nm-silicon-oxide case, we set the laser intensity at $\mathrm{ED}=700 \mathrm{~mJ} / \mathrm{cm}^{2}$. The evolution of the thermal fields and moving fronts during the melting and solidification processes is shown in Figs. 9 and 10, respectively. During the melting process, because the heat dissipation rate on the substrate and $a$-Si film was higher at the edge, the interface shape was curved, while the central front $(\sim 1 \mu \mathrm{m})$ was almost planar similar to the 1D case. When the planar interface reached the oxide, the rapid heat accumulation drove the lateral fronts outward. Gradually, the melted region exceeded the width of the irradiation slit; in this case, it exceeded the width by about $0.1 \mu \mathrm{m}$ on each side. When the laser pulse terminated, the interfaces became almost vertical, and recrystallization was triggered, as the one (60 ns) shown in Fig. 10.

As mentioned in the previous section, the vertical growth rate of FGs was about $2 \mathrm{~m} / \mathrm{s}$ here due to the same direction of heat release and dissipation. For lateral growth, many simulation studies indicated that the solidification speed was on the order of $10 \mathrm{~m} / \mathrm{s} .{ }^{11,15}$ Because of the higher thermal conductivity of molten $\mathrm{Si}$, a large amount of heat is concentrated in the central part of the irradiation region, and the direct contact between the oxide and molten $\mathrm{Si}$ also as- 

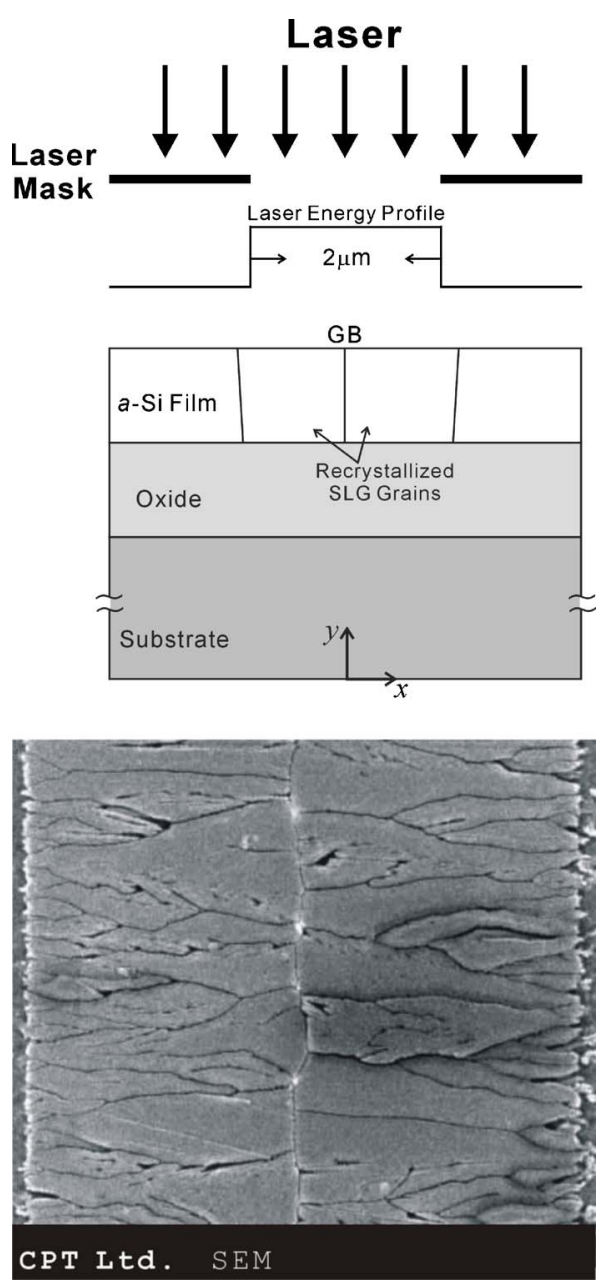

FIG. 8. (a) Schematic diagram of a unit of the simplified sequential lateral solidification (SLS) system and the laser beam profile, where GB is the grain boundary, resulting from conflict between SLG grains approaching from two sides; (b) top view of a sample experimentally observed grain structure (following Secco solution etching).

sists heat dissipation. As a result, greater supercooling occurs on the interface, leading to fast solidification. In addition, as shown in Fig. 10, during the process, the fronts stay almost unchanged and the thermal fields remain uniform in the vertical direction. Therefore, we conclude that recrystallized SLG film obtained by using SLS is quite uniform in this direction, unlike the FG one from the vertical growth.

For crystal growth, a uniform thermal field and growth speed during crystallization are very important and especially affect the distribution of defects. ${ }^{31}$ To demonstrate the lateral uniformity of SLS films, the history of the center temperature at the bottom of the silicon film is shown in Fig. 11. The insert in Fig. 11 shows the lateral temperature profiles at various times extracted from the bottom portion of the $\mathrm{Si}$ film. During the initial stage of crystallization, the temperature at the central point decreased rapidly with time, but the temperature of the molten $\mathrm{Si}$ exceeded that of the interface, as shown by the curve of $70 \mathrm{~ns}$ in Fig. 11. Gradually, the center temperature fell below the interface temperature, and the effect of latent heat release was clearly observed (e.g., the curve at $116 \mathrm{~ns}$ ). During the final stage, because the two grains were approaching each other, the thermal boundary
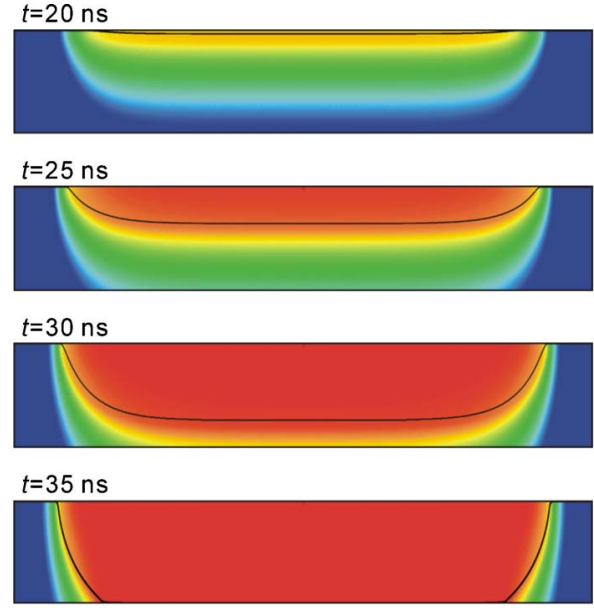

$t=40 \mathrm{~ns}$
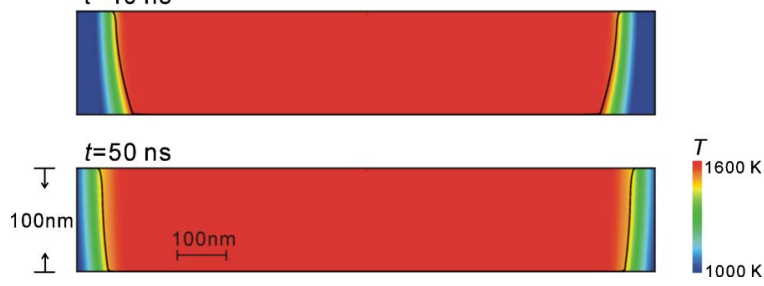

FIG. 9. (Color online) Simulated positions of the $a$-Si/melt interface $(\phi$ $=0.5$ ) and the thermal field during the melting process following laser irradiation of $700 \mathrm{~mJ} / \mathrm{cm}^{2}$. Note that the $y$ direction is rescaled.

layers bunched together so that the center temperature increased. At $160 \mathrm{~ns}$, the temperature profile of molten Si was almost uniform. On the other hand, the merging of the thermal boundary layers caused both center and interface temperatures to increase.
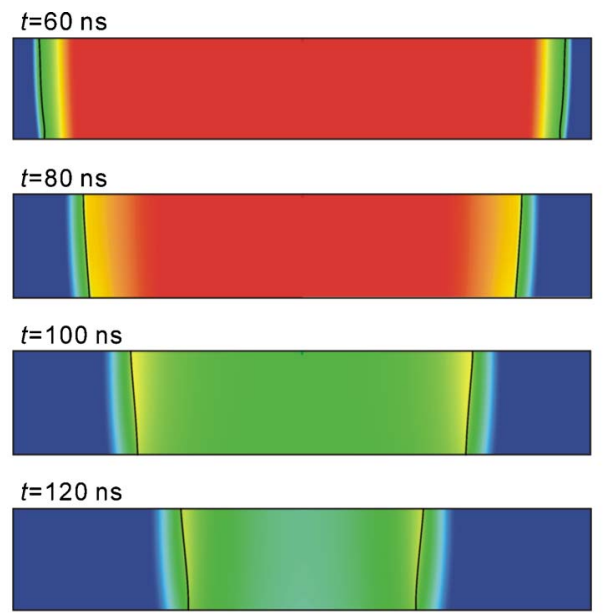

$t=140 \mathrm{~ns}$
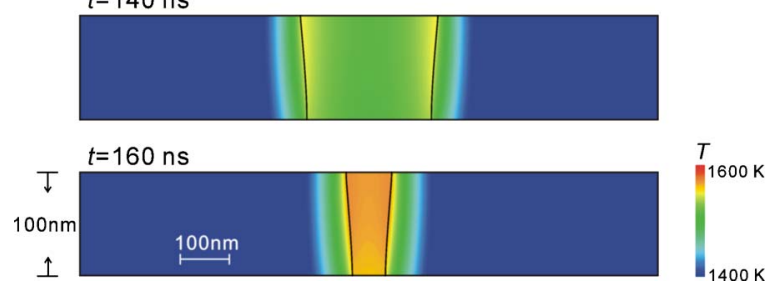

FIG. 10. (Color online) Simulated positions of the SLG-Si/melt interface $(\phi=0.5)$ and the thermal fields during the recrystallization process. Note that the $y$ direction is rescaled. 


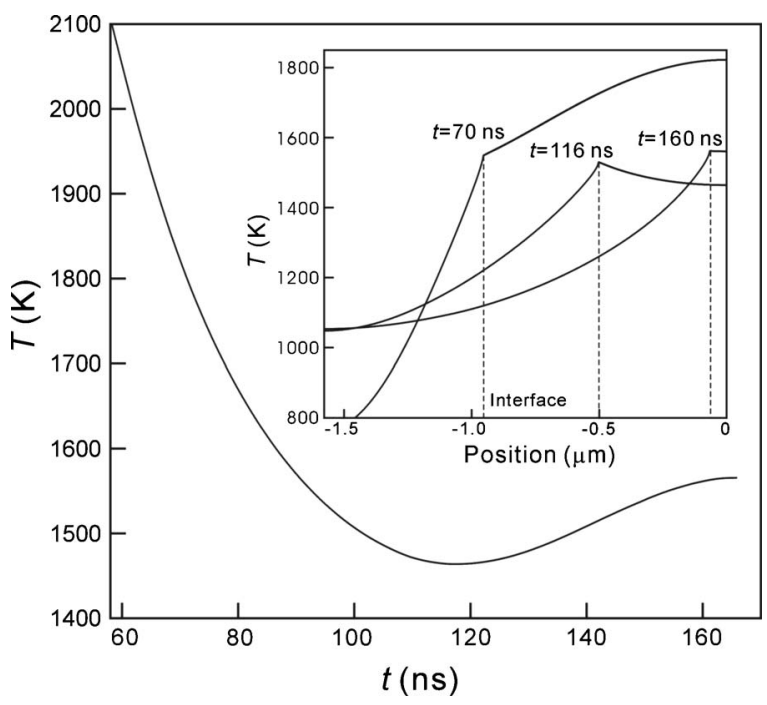

FIG. 11. Calculated central temperature $(x=0)$ at the bottom of the Si film. The temperature profiles at various times are also shown in the upper-right portion of the figure.

Interestingly, according to the above analysis, because several thermal effects occur along with the propagation of the interfaces, uniform lateral growth of SLS grains seems to be impossible to achieve. The interface temperature and solidification speed during the growth process based on the position of the recrystallized grain are plotted in Fig. 12. As shown, the grain structure could be roughly divided into three regions. During the onset of crystallization, fast growth released a large amount of heat, so that the interface temperature increased, thus slowing down solidification. During this stage, because the rate of heat rejection was much faster than that of the diffusion, overshoot of the interface temperature could occur. Then, with the further decrease of the center temperature, both the heat diffusion and growth speed increased, as shown in Fig. 12; the grain-developing region in this case was about $0.5 \mu \mathrm{m}$ on each side. In practice, this transformation structure can be eliminated by a second laser shot treatment.

The continuous decrease in the center temperature implies that a steady growth speed was not achieved until the thermal boundary layers on the two sides came to contact. As explained above, the effect of latent heat release from both sides of the fronts stopped the reduction in the center temperature (around 110-120 ns in Fig. 11), while the rates of

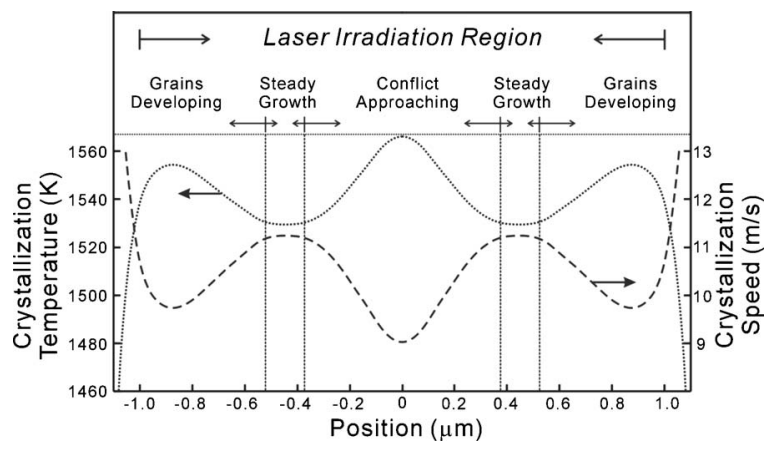

FIG. 12. Simulated crystallization temperature and speed during growth based on the position of the fabricated $p$-Si film. heat rejection and thermal dissipation reached quasisteady states. Accordingly, a steady growth region existed, but it was relatively small, about $0.1-0.2 \mu \mathrm{m}$ in size. When two grains were approaching each other, the crystallization temperature increased, thus slowing down solidification. The minimum growth speed occurred at the center of the laser shot $(\sim 9 \mathrm{~m} / \mathrm{s})$, and this corresponded to the highest interface temperature of about $1570 \mathrm{~K}$ in the present case. In fact, due to the higher crystallization temperature in the central part of the laser shot, the density of defects here may be lower. Previous experiments ${ }^{32}$ have shown that the devices located here have a better electric reliability under hot-carrier stress, although a conflicting grain boundary appears. In short, our study clearly indicates that due to the instability of SLG, to obtain uniform electric characteristics, the layout design for the dimensions and the positions of devices should take the SLG laser mask into consideration.

\section{CONCLUSIONS}

In this paper, we have presented a phase field model that can simulate laser crystallization processing of a silicon film on an amorphous substrate. The phase field and thermal equations have been derived directly from the coupled Gibbs-Thompson relation and the energy conservation. For one-dimensional cases, the nonequilibrium behavior of the interface movement speed and temperature has been confirmed. The melt depth and duration under various energy densities have been determined, and the overall trend is consistent with those obtained in previous experiments. Twodimensional simulations of the SLG phenomena have been conducted. The detailed evolution of the thermal and phase fields has been clearly illustrated, and some important results have been obtained. Because the interface and transport properties are close to the real situation, the phase field simulation studies should be useful for optimizing the laser annealing process. In the future, both kinetic and capillary anisotropic effects will be embedded in real three-dimensional simulations. If the computational load can be reduced, the modeling of texture-controlled lateral growth will apparently be possible.

\section{ACKNOWLEDGMENTS}

The authors gratefully thank Dr. Antonino La Magna for his kind explanation of his phase field modeling of the laser annealing process, and thank the Japan Steel Works, Ltd. for fabricating the SLS samples used in this work. The support from the LTPS group in CPT is gratefully acknowledged.

${ }^{1}$ T. Matsuo and T. Muramatsu, SID Int. Symp. Digest Tech. Papers 2004, 856.

${ }^{2}$ H. Kuriyama, T. Nohda, S. Ishida, T. Kuwahara, S. Noguchi, S. Kiyama, S. Tsuda, and S. Nakano, Jpn. J. Appl. Phys., Part 1 32, 6190 (1993).

${ }^{3}$ J. S. Im, H. J. Kim, and M. O. Thompson, Appl. Phys. Lett. 63, 1969 (1993).

${ }^{4}$ J. S. Im and H. J. Kim, Appl. Phys. Lett. 64, 2303 (1994).

${ }^{5}$ R. S. Sposili and J. S. Im, Appl. Phys. Lett. 69, 2864 (1996).

${ }^{6}$ H. J. Kim and J. S. Im, Appl. Phys. Lett. 68, 1513 (1996).

${ }^{7}$ H. Ogawa, M. Hiramatsu, Y. Kimura, M. Jyumonji, Y. Taniquchi, and M. Matsumura, in Proceedings of the 10th International Display Workshop Conference (SID, Japan, 2003), pp. 323-326.

${ }^{8}$ I. H. Song, S. H. Kang, J. H. Kim, and M. K. Han, SID Int. Symp. Digest 
Tech. Papers 2003, 1310.

${ }^{9}$ M. O. Thompson, G. J. Galvin, J. W. Mayer, P. S. Peercy, J. M. Poate, D. C. Jacobson, A. G. Cullis, and N. G. Chew, Phys. Rev. Lett. 52, 2360 (1984).

${ }^{10}$ R. F. Wood and G. A. Geist, Phys. Rev. B 34, 2606 (1986).

${ }^{11}$ R. F. Wood, G. A. Geist, and C. L. Liu, Phys. Rev. B 53, 15863 (1996)

${ }^{12}$ J. P. Leonard and J. S. Im, Appl. Phys. Lett. 78, 3454 (2001).

${ }^{13}$ R. Černý and P. Přikryl, Phys. Rev. B 57, 194 (1998).

${ }^{14}$ V. V. Gupta, H. J. Song, and J. S. Im, Appl. Phys. Lett. 71, 99 (1997).

${ }^{15}$ H. Kisdarjono, A. T. Voutsas, and R. Solanki, J. Appl. Phys. 94, 4374 (2003).

${ }^{16}$ R. F. Wood and G. E. Giles, Phys. Rev. B 23, 2923 (1981).

${ }^{17}$ W. J. Boettinger, J. A. Warren, C. Beckermann, and A. Karma, Annu. Rev. Mater. Res. 32, 163 (2002).

${ }^{18}$ A. Karma and W. J. Rappel, Phys. Rev. E 53, R3017 (1996).

${ }^{19}$ A. Karma, Phys. Rev. Lett. 87, 115701 (2001).

${ }^{20}$ C. W. Lan, C. J. Shih, and M. H. Lee, Acta Mater. 53, 2285 (2005).

${ }^{21}$ N. A. Ahmad, A. A. Wheeler, W. J. Boettinger, and G. B. McFadden, Phys. Rev. E 58, 3436 (1998).

${ }^{22}$ N. Provatas, N. Goldenfeld, and J. Dantzig, Phys. Rev. Lett. 80, 3308
(1998).

${ }^{23}$ C. W. Lan, C. C. Liu, and C. M. Hsu, J. Comput. Phys. 178, 464 (2002).

${ }^{24}$ A. La Magna, P. Alippi, V. Privitera, G. Fortunato, M. Camalleri, and B. Svensson, J. Appl. Phys. 95, 4806 (2004).

${ }^{25}$ A. La Magna, P. Alippi, V. Privitera, S. Scalese, S. Pannitteri, G. Fortunato, L. Mariucci, and M. Camalleri, Appl. Phys. Lett. 84, 4738 (2004).

${ }^{26}$ A. La Magna, P. Alippi, V. Privitera, and G. Fortunato, Appl. Phys. Lett. 86, 161905 (2005).

${ }^{27}$ C. Beckermann, H. J. Diepers, I. Steinbach, A. Karma, and X. Tong, J. Comput. Phys. 154, 468 (1999).

${ }^{28}$ S. R. Stiffler, P. V. Evans, and A. L. Greer, Acta Metall. Mater. 40, 1617 (1992).

${ }^{29}$ J. H. Jeong, N. Goldenfeld, and J. A. Danzig, Phys. Rev. E 64, 041602 (2001).

${ }^{30}$ J. S. Im et al., Phys. Status Solidi A 166, 603 (1998).

${ }^{31}$ V. V. Voronkov, J. Cryst. Growth 59, 625 (1982).

${ }^{32}$ H. T. Chen, Y. C. Chen, P. H. Tsai, J. X. Lin, C. L. Chen, and C. J. Chang, in Proceedings of International Display Manufacturing Conference '05 (SID, Taiwan, 2005), pp. 55-58. 